\title{
Using of Network Motifs on Basketball Tactical Analysis
}

\author{
Halil Orbay Çobanoğlu \\ Correspondence: Halil Orbay Çobanoğlu, Muzaffer Çil Anadolu High School, Ministry of National Education, \\ Eskişehir, Turkey.
}

Received: January 28, 2019

Accepted: February 17, 2019

Online Published: February 26, 2019

doi:10.11114/jets.v7i3S.4105

URL: https://doi.org/10.11114/jets.v7i3S.4105

\begin{abstract}
In the specific case of team sports there are a particular interest to recognize the cooperation tendencies between team-members. There are several ways to extend the investigation of pass motifs to reveal finergrained details of teams and players. The aim of this study was to solve the game strategies of basketball teams by using network science through motifs. The study sample consists of 6 matches of Turkish National Basketball Team in the EuroBasket2015 tournament. 6 matches were analyzed with e-analysis basketball program, the pass actions obtained from the analysis were transferred into Excel files. The Excel pass files were processed with the open-source program Gephi to obtain the network of the matches and the measurements of networks' motifs. And then using R and R package igraph 3-nodes and 4-nodes pass motifs were found. As a result, for 3-nodes Type 9 has been seen as mode 2 times. With the help of network science approach and by using motifs the most influential players can be found, the most compatible squad for future games can be selected and the opponent's key players can be analyzed. If coaches want to disrupt the opponent's game format, they must first be aware of the pass motifs that the team often uses. Determining how to break these motifs will make an important contribution to the success of a team.
\end{abstract}

Keywords: network science, motifs, basketball, tactics

\section{Introduction}

Complex networks have been studied in many disciplines (Milo, Shen-Orr, Itzkovitz, Kashtan, Chklovskii \& Alon, 2002). Many complex networks share global features such as power-law degree distribution and the small world property. Nowadays, observations of biological networks revealed the existence of network motifs: small connected subgraphs occurring significantly higher than in randomized networks with the same degree sequence (Heinlein, 2019). The motifs in a network are small connected subnets formed at much higher frequencies than random networks (Li, Xiao, Zhou \& Yang, 2012). Recently, they have received a lot of attention as a useful concept to reveal the structural design principles of complex networks (Kashtan, Itzkovitz, Milo \& Alon, 2004; Wernicke, 2005). A network is a set of vertices, sometimes called nodes, with links between them, called edges. A complex network is a network characterized by a large number of vertices and edges that follow some pattern, like the formation of clusters or highly connected vertices, called hubs. While in a simple network, with at most hundreds of vertices, the human eye is an analytic tool of remarkable power, in a complex network, this approach is useless. Therefore, to examine complex networks, we need to use statistical methods to tell us how the network looks (Vaz de Melo, Almeida \& Loureiro, 2008). Capturing the interaction between individuals within a group is a central purpose of network analysis. Useful illustrations of the network structure should provide information about the purpose and functionality of networks (Fewel, Armbruster, Ingraham, Petersen \& Waters, 2012). Network science gives information about the cliques and motifs that dominate the game. For example, with closeness centrality, how well connected a player to the team can be calculated. Out-degree of a player can be used as a metric tomeasure the passes he has given. The clustering coefficient can be used as a sign of "possession" in the pass network. And, betweenness, which naturally capture the hubs and essential associations in the distribution of the ball, has crucial importance to analyze network metrics (Cotta, Mora, Merelo-Molina \& Merelo, 2011).

Nowadays, sport is now considered as a complex networks often display network motifs and these can be described as subgraphs that recur in the network much more often than in randomized networks (Gürsakal, Yılmaz, Çobanoğlu \& Çağliyor, 2018). Performance analysis in team sports aims to measure and process data from training sessions and competitions with a view to use the information to improve future performances (Sampaio, Leser, Baca, Calleja-Gonzalez, Coutinho, Gonçalves \& Leite, 2016). Different computer-based approaches have been attempting to extract and analyse tactical patterns in team sports (Clemente, Couceiro, Martins \& Mendes, 2015). Basketball is a complex and dynamic 
system based on interactions. These interactions within a team can be considered as a collaborative process based on the strategic plan, the state variables and contextual constraints for the team (Clemente, Martins, Kalamaras, Oliveira, Oliveira \& Mendes (2015). The use of data mining techniques provides information about the individual qualities and habits of players in both attack and defense; therefore, it is easier to predict where the advantages of problems will occur in individual offense or defense situations. With this in mind, a coach can pay more or less attention to a particular part of a game, reduce the number of information, and give all players of the team an explicit and identical idea of how to play this game (Markoski, Ivankovic \& Ivkovic, 2012).

\subsection{Number of Motifs and Network Types}

Motifs are small, local, patterns of interconnections that occur throughout a network with significantly higher probability than in random networks. In other words, network motifs are small connected subnetworks within a larger network that occur in statistically significant quantities and may indicate functional regions of the network. Network motif software tools employ algorithms that compare a network to randomly generated networks in order to identify subnetworks that occur in frequencies higher than would be expected by random chance (Luciano, 2009). Network motif identification is significant in that motifs generally reflect functionalities. However, the task is greatly challenging due to diverse patterns of motifs possibly existent in a network and high computation complexity for large scale networks (Zhang \& Xue, 2013). These basic structural elements are the building blocks that make up the network and are crucial for understanding how the network functions (Huang, Murray, Shen, Song, Wu \& Zheng, 2005). Owing to their small size, motifs can be explicitly identified and enumerated in various networks, each network being characterized by its own set of distinct motifs (Dobrin, Beg, Barabási \& Oltvai, 2004).

Table 1. Number of motifs according to network types

\begin{tabular}{cccc}
\hline Network type & 3 nodes & 4 nodes & 5 nodes \\
\hline Undirected & 2 & 6 & 21 \\
Directed & 13 & 199 & 9364
\end{tabular}

As it can be seen from Table 1, for undirected networks, number of 3-nodes motifs are 2 (Figure 1) (Gürsakal et al., 2018).

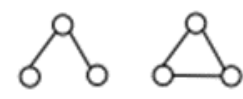

Figure 1. 2 undirected 3-nodes motifs

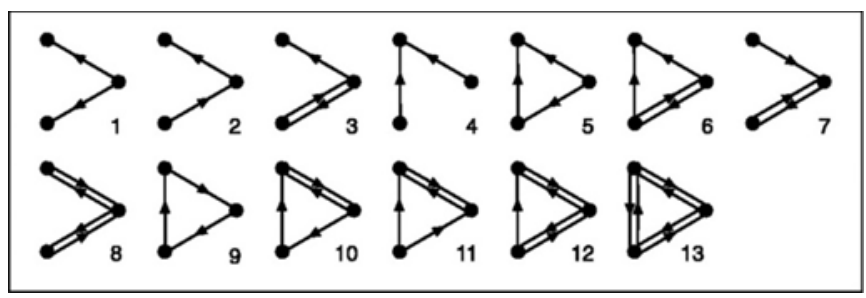

Figure 2. 13 directed 3-nodes motifs

Also 13 directed 3-nodes motifs can be seen in Figure 2 above (Gürsakal et al., 2018).

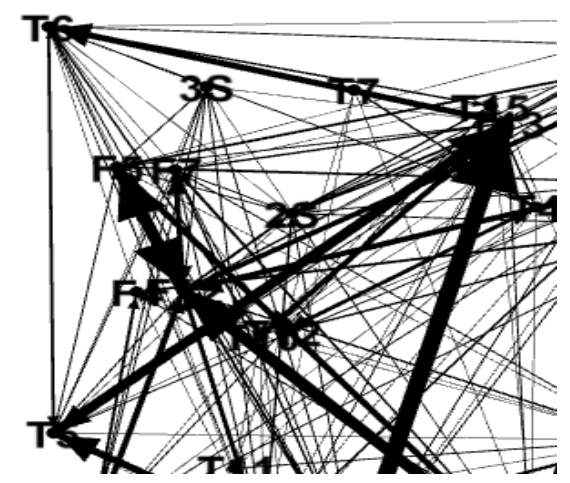

Figure 3. Motifs from France-Turkey Match (12-09-2015)

Figure 3 shows us that 3-node motifs like T5-T6-T15 (This is 13th motif in Figure 2). 


\section{Methods}

The study sample consists of 6 matches of Turkish National Basketball Team in the EuroBasket2015 tournament. 6 matches were analyzed with e-analysis basketball program, the pass actions obtained from the analysis were transferred into Excel files. The Excel pass files were processed with the open-source program Gephi to obtain the network of the matches and the measurements of networks' motifs.

\section{Results}

\subsection{Basketball Motifs}

In this study 6 National Teams matches' data were used and these matches can be seen in Table 3. Using R and R package igraph 3-nodes and 4-nodes pass motifs were found. As an example let's take a match between France and Turkey and apply the codes below for the Turkish team.

$>$ View(fratur2ags)

$>$ library(readxl)

> fratur2ags1 <- read_excel("C:/Users/LENOVO/Desktop/basketbolyeniveri/fratur2ags1.xlsx")

$>$ View(fratur2ags1)

$>$ View(fratur2ags1)

$>$ library('igraph')

$>$ network <- graph_from_data_frame $(\mathrm{d}=$ fratur2ags 1 , directed=T)

$>$ plot(network)

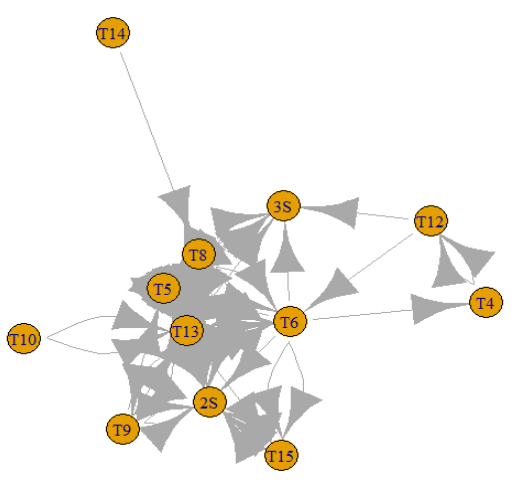

Figure 4. Pass network of the Turkish National Basketball Team

$>$ motif $=$ graph. motifs(network,size $=3$ )

$>$ motif

[1] NA NA 10 NA 138118052111031

$>\operatorname{plot}($ graph. isocreate $($ size $=3$, number $=2)$ )

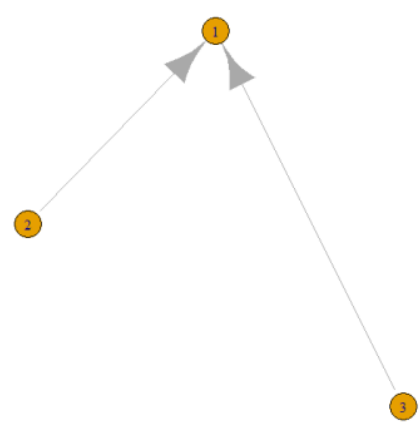

Figure 5. The 3 node motif of The Turkish team

$>$ motif=graph.motifs(network,size $=4$ )

$>$ motif 


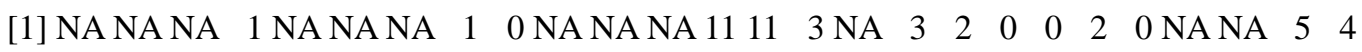

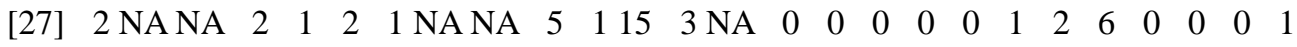

[53] $0 \begin{array}{llllllllllllllllllllllllll}0 & 0 & 0 & 0 & 4 & 1 & 2 & 0 & 2 & 0 & \mathrm{NA} & 0 & 2 & 2 & 0 & 0 & 0 & 0 & 0 & 0 & 0 & 0 & 0 & 0 & 3 & 6\end{array}$

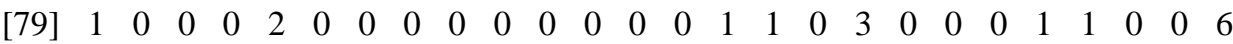

[105] $10 \begin{array}{llllllllllllllllllllllllll} & 0 & 0 & 3 & 1 & 0 & 0 & 0 & 0 & 0 & 0 & 3 & 0 & 2 & 1 & 0 & \mathrm{NA} & 0 & 0 & 2 & 1 & 0 & 0 & 0 & 0 & 0\end{array}$

[131] $00 \begin{array}{llllllllllllllllllllllllll} & 0 & 0 & 0 & 0 & 0 & 0 & 0 & 1 & 1 & 0 & 0 & 0 & 1 & 0 & 0 & 0 & 0 & 0 & 0 & 0 & 0 & 2 & 0 & 0 & 0\end{array}$

[157] $0 \begin{array}{lllllllllllllllllllllllllll}0 & 0 & 0 & 0 & 0 & 0 & 0 & 0 & 0 & 0 & 0 & 1 & 0 & 0 & 0 & 0 & 0 & 2 & 0 & 0 & 5 & 1 & 0 & 0 & 0 & 0\end{array}$

[183] $0 \begin{array}{llllllllllllllllllllllllll}0 & 0 & 1 & 0 & 5 & 0 & 0 & 0 & 0 & 0 & 0 & 0 & 0 & 0 & 0 & 0 & 0 & 0 & 0 & 0 & 0 & 0 & 0 & 0 & 0 & 0\end{array}$

[209] $00 \begin{array}{lllllllll}0 & 0 & 0 & 1 & 2 & 0 & 0 & 0 & 0\end{array}$

$>\operatorname{plot}($ graph.isocreate $($ size $=4$, number $=22))$

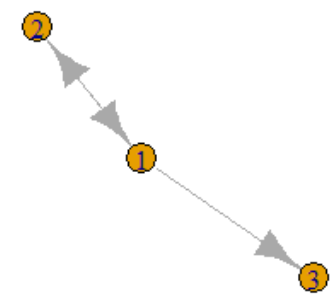

Figure 6. The 4 node motif of The Turkish team 
Table 2. Matches and Turkey Networks

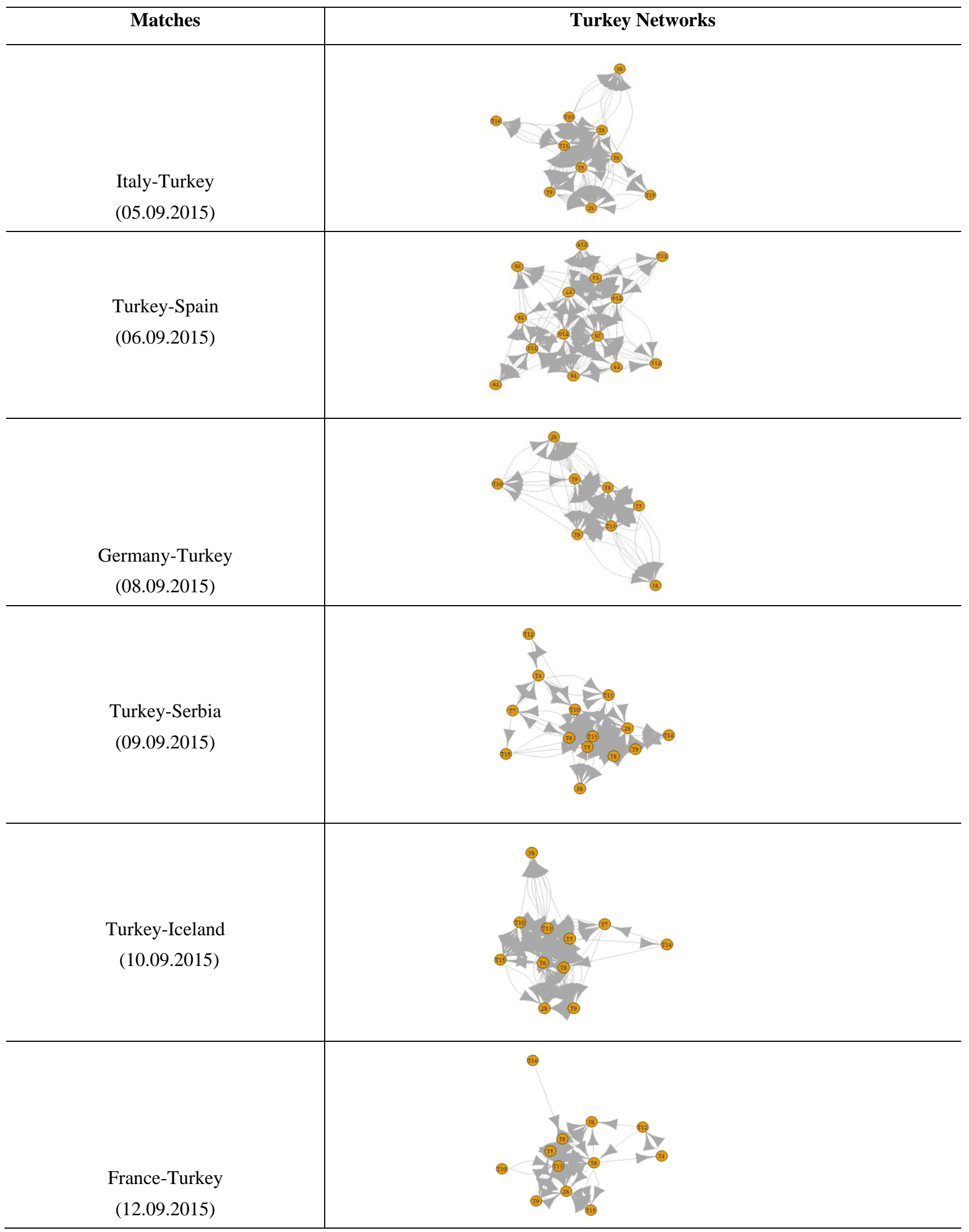


Table 3. 3-nodes motifs results

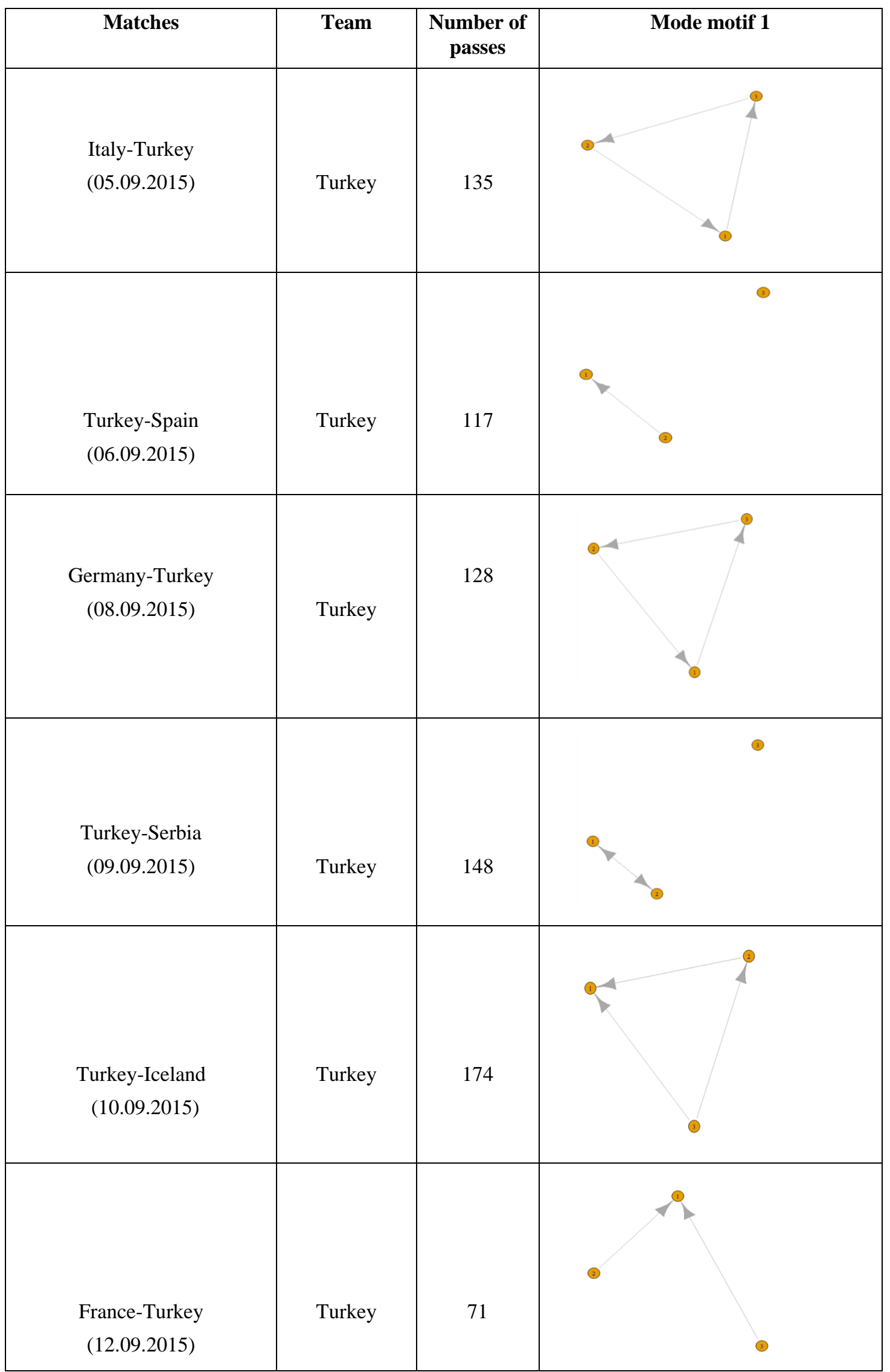


As a result, in Table 3 for 3-nodes considering Figure 2, type 9 has been seen as mode 2 times.

Table 4. 4-nodes motifs results

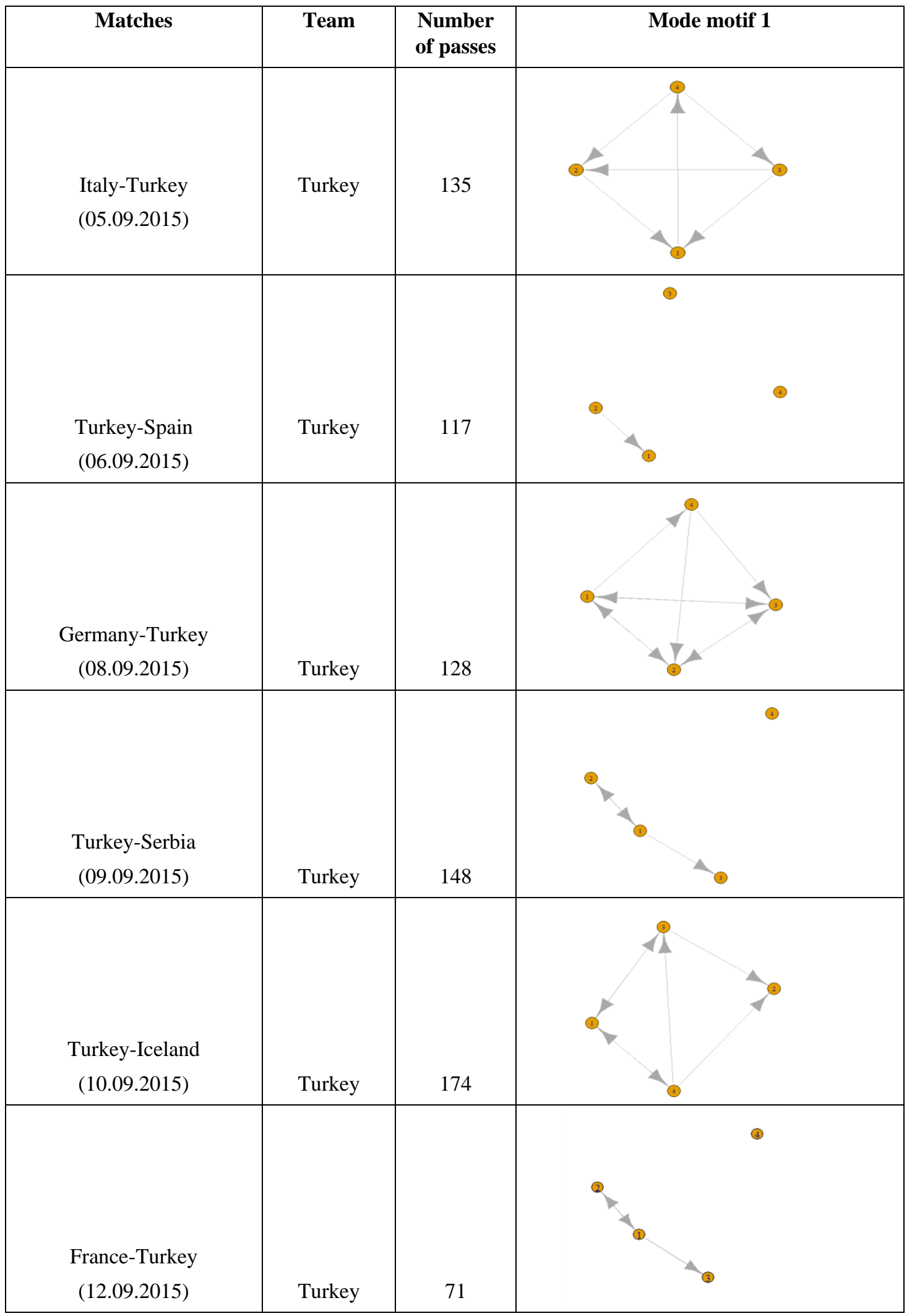

As a result, in Table 4 for 4-nodes considering Figure 6 has been repeated 2 times between 6 motifs. 


\section{Discussion and Conclusion}

The aim of this study was to solve the game strategies of basketball teams by using network science through motifs. In this research, it was determined that which 3-nodes and 4-nodes motif types were the mod motif types within the framework of a 6-match data set. As a result, in Table 3 for 3-nodes considering Figure 2, type 9 has been seen as mode 2 times and in Table 4 for 4 -nodes considering Figure 6 has been repeated 2 times between 6 motifs. Clemente, Martins, Kalamaras \& Mendes (2015) in their study which aim was to identify the centrality tactical positions in basketball they emphasized that using the social network analysis metrics was possible to conclude that point guard is the prominent tactical position that links the remaining team-members during the attacking process and it was observed no differences between different competitive leagues considering the centrality levels. Clemente, Martins \& Mendes (2015) in their study which aim was to analyze the variance of general network metrics between different team sports and competitive levels and they found that team sports with greater number of players increased the values of total arcs and team sports with smaller players increases the density values and the clustering coefficient, thus suggesting the better values of cohesion emerges in smaller formats. Vaz de Melo et al., (2008) in their study they examined that the evolution of the NBA using complex network metrics, calculated from a graph in which the vertices are the players and the teams, and the edges identify labor relationships among them. And they estimated that in their study they showed the evolution and growth of the NBA social network, constructed from data obtained in the NBA database and showed the NBA network structure can be characterized as a small-world network. They also predicted that complex network metrics could be used to analyze social relationships between NBA players and discover new information in the NBA database. In addition, this new kind of information derived from network relationships has been improved in understanding team behavior (Vaz de Melo et al., 2008). Fewel et al. (2012) in their study asked that how team dynamics can be captured in relation to function by considering games as networks. They defined players as nodes and ball movements as links, they analyzed the network properties of degree centrality, clustering, entropy and flow centrality across teams and positions, to characterize the game from a network perspective and to determine whether they could assessed differences in team offensive strategy by their network properties. Their analysis showed the utility of network approaches in quantifying team strategy and showed that testable hypotheses could be evaluated using this approach and also highlight the richness of basketball networks as a dataset for exploring the relationships between network structure and dynamics with team organization and effectiveness (Fewel et al., 2012). In the other study authors used a graph theory to analyse the relationship between basketball players in each unit of attack crossing this quantitative analysis with a qualitative one to explain social interactions. They found the rise of a specific network regarding each team. These results suggest that the networks' coordinations were built on local interactions that do not necessarily require all players to achieve the team's goal (Bourbousson, Poizat, Saury \& Carole, 2010). Kooij, Jamakovic, Kesteren, Koning, Theisler \& Veldhoven (2009) in their study emphasized that by using network science coaches could be determined the optimal line-up for matches. In other study Piette, Pham \& Anand (2011) studied on network science in sports and they emphasized that to the importance of how two teammates perform together. And in this way they performed the same type of performance evaluation on pairs of teammates, which could highlight players who while not successful individually, work great as a couple. Gürsakal, Çobanoğlu \& Çağlıyor (2017) in their study emphasized that with the help of network science approach, the most influential players can be found, the most compatible squad for future games can be selected and the opponent's key players can be analyzed.

If coaches want to disrupt the opponent's game format, they must first be aware of the pass motifs that the team often uses. Determining how to break these motifs will make an important contribution to the success of a team. Examination of the mode pass types of the teams that are very successful and consistently winning is important for the other teams who want to achieve this success. The most important deficiency of the pass network analysis method is that this analysis does not take into account the position of the players in the field in any way.

\section{References}

Bourbousson, J., Poizat, G., Saury, J., \& Carole, S. (2010). Team Coordination in Basketball: Description of the Cognitive Connections Among Teammates. J Appl Sport Psychol, 22(2), 150-166. https://doi.org/10.1080/10413201003664657

Clemente, F. M., Couceiro, M. S., Martins, F. M. L., \& Mendes, R. S. (2015). Using network metrics in soccer: a macro-analysis. Journal of Human Kinetics, 45, 123-134. https://doi.org/10.1515/hukin-2015-0013

Clemente, F. M., Martins, F. M. L., \& Mendes, R. S. (2015). How team sports behave as a team? General network metrics applied to sports analysis. Sport Science, 8(Suppl 2), 81-87.

Clemente, F. M., Martins, F. M. L., Kalamaras, D., \& Mendes, R. S. (2015). Network analysis in basketball: inspecting the prominenet players using centrality metrics. Journal of Physical Education and Sport, 15(2), 212-217.

Clemente, F. M., Martins, F. M. L., Kalamaras, D., Oliveira, J., Oliveira, P., \& Mendes, R. S. (2015). The social network 
analysis of Switzerland Football Team on Fifa World Cup 2014. Acta Kinesiologica, 9(1), 25-30.

Cotta, C., Mora, A. M., Merelo-Molina, C., \& Merelo, J. J. (2011). FIFA World Cup 2010: A Network Analysis of the Champion Team Play, arXiv:1108.0261v1 [cs.SI].

Dobrin, R., Beg, Q. K., Barabási, A. L., \& Oltvai, Z. N. (2004). Aggregation of topological motifs in the Escherichia coli transcriptional regulatory network. BMC bioinformatics, 5, 10. https://doi.org/10.1186/1471-2105-5-10

Fewel, J. H., Armbruster, D., Ingraham, J., Petersen, A., \& Waters, J. S. (2012). Basketball teams as strategic networks. Plos One, 7(11), e47445. https://doi.org/10.1371/journal.pone.0047445

Gürsakal, N., Çobanoğlu, H. O., \& Çağlıyor, S. İ. (2017). A network science approach: How different are the attacks of football? Pamukkale Journal of Sport Science, 8(3), 45-57.

Gürsakal, N., Yılmaz, F. M., Çobanoğlu, H. O., \& Çağlıyor, S. İ. (2018). Network Motifs in Football. Turkish Journal of Sport and Exercise, 20(3), 263-272. https://doi.org/10.15314/tsed.465664

Heinlein, A. (2019, February 16). Network Analyzation and Optimization using Network Motifs. Retrieved from http://choerbaert.org/scy/stud/hs_telematik/tF03_txt.pdf

Huang, W., Murray, C., Shen, X., Song, L., Wu, Y. X., \& Zheng, L. (2005). Visualisation and analysis of network motifs. Ninth International Conference on Information Visualisation (IV'05), 697-702. https://doi.org/10.1109/IV.2005.138

Kashtan, N. Itzkovitz, S., Milo, R., \& Alon, U. (2004). Efficient sampling algorithm forestimating subgraph concentrations and detecting network motifs. Bioinformatics, 20(11), 1746-1758. https://doi.org/10.1093/bioinformatics/bth163

Kooij, R., Jamakovic, A., Kesteren, F. V., Koning, T. D., Theisler, I., \& Veldhoven, P. (2009). The Dutch Soccer Team as a social network. Retrieved from file:///C:/Users/LENOVO/Downloads/kooij-2009-dutch.pdf (24.01.2019).

Li, J., Xiao, F., Zhou, J., \& Yang, Z. (2012). Motifs and Motif Generalization in Chinese Word Networks. Procedia Computer Science, 9, 550-556. https://doi.org/10.1016/j.procs.2012.04.059

Luciano, C. (2009). Network Motifs and E. coli. Retrieved from https://pdfs.semanticscholar.org/a496/3454b8bc8d036b744e0608ab6998afb3a820.pdf

Markoski, B., Ivankovic, Z., \& Ivkovic, M. (2012). Using neural networks in preparing and analysis of basketball scouting. Retrieved from http://dx.doi.org/10.5772/48178.

Milo, R., Shen-Orr, S., Itzkovitz, S., Kashtan, N., Chklovskii, D., \& Alon, U. (2002). Networks motifs: simple building blocks of complex networks. Science, 298(5594), 824-7. https://doi.org/10.1126/science.298.5594.824

Piette, J., Pham, L., \& Anand, S. (2011). Evaluating basketball Player Performance via Statistical Network Modeling. MIT Sloan Sports Analytics Conference, March 4-5, Boston, MA, USA.

Sampaio, J., Leser, R., Baca, A., Calleja-Gonzalez, J., Coutinho, D., Gonçalves, B., \& Leite, N. (2016). Defensive pressure affects basketball technical actions but not the time-motion variables. J Sport Health Sci., 5(3), 375-380. https://doi.org/10.1016/j.jshs.2015.01.011

Vaz de Melo, P. O. S., Almeida, V. A. F., \& Loureiro, A. A. F. (2008). Can complex network metrics predict the behaviour of NBA teams? KDD 08, August24-27, Las Vegas, Nevada, USA.

Wernicke, S. (2005). A faster algorithm for detecting network motifs. WABI, 3692, 165-177. https://doi.org/10.1007/11557067_14

Zhang, J., \& Xue, Y. (2013). Motif Identification Based on Local Structure Clustering. In: Yin Z., Pan L., Fang X. (eds), Proceedings of The Eighth International Conference on Bio-Inspired Computing: Theories and Applications (BIC-TA), 2013. Advances in Intelligent Systems and Computing, (pp. 361-367), 212. Springer, Berlin, Heidelberg. https://doi.org/10.1007/978-3-642-37502-6_44

\section{Copyrights}

Copyright for this article is retained by the author(s), with first publication rights granted to the journal.

This is an open-access article distributed under the terms and conditions of the Creative Commons Attribution license which permits unrestricted use, distribution, and reproduction in any medium, provided the original work is properly cited. 